\title{
Evaporative Cooling and Dehumidification Garment for Portable Life Support Systems
}

\author{
Michael Izenson ${ }^{1}$ and Weibo Chen ${ }^{2}$ \\ Creare Inc., Hanover, NH, 03755 \\ Grant Bue ${ }^{3}$ \\ NASA Lyndon B. Johnson Space Center, Houston, Texas, 77062
}

\begin{abstract}
This paper describes the design and development of an innovative thermal and humidity control system for future space suits. The system comprises an evaporation cooling and dehumidification garment (ECDG) and a lithium chloride absorber radiator (LCAR). The ECDG absorbs heat and water vapor from inside the suit pressure garment, while the LCAR rejects heat to space without venting water vapor. The ECDG is built from thin, flexible patches with coversheets made of non-porous, water-permeable membranes that -enclose arrays of vapor flow passages. Water vapor from inside the spacesuit diffuses across the water permeable membranes, enters the vapor flow channels, and then flows to the LCAR, thus dehumidifying the internal volume of the space suit pressure garment. Additional water evaporation inside the ECDG provides cooling for sensible heat loads. -The heat released from condensation and absorption in the LCAR is rejected to the environment by thermal radiation. We have assembled lightweight and flexible ECDG pouches from prototypical materials and measured their performance in a series of separate effects tests under well-controlled, prototypical conditions. Sweating hot plate tests at typical space suit pressures show that ECDG pouches can absorb over $60 \mathrm{~W} / \mathrm{ft}^{2}$ of latent heat and $20 \mathrm{~W} / \mathrm{ft}^{2}$ of sensible heat from the pressure garment environment. These results are in good agreement with the predictions of our analysis models.
\end{abstract}

\section{Nomenclature}

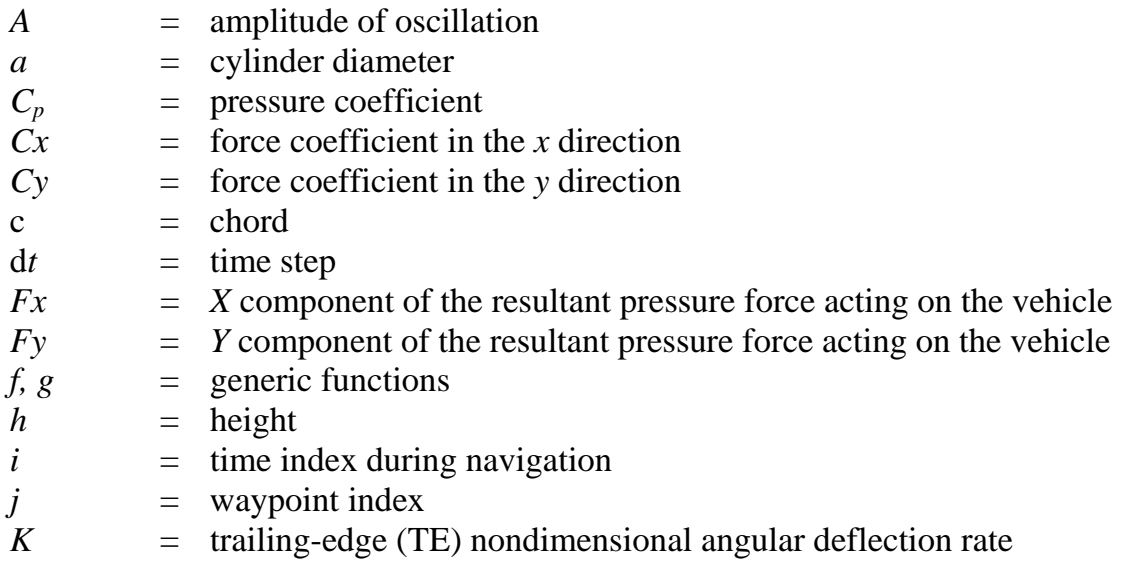

\footnotetext{
${ }^{1}$ Principal Engineer, 16 Great Hollow Road, Hanover, NH, 03755, Senior Member of AIAA..

${ }^{2}$ Senior Engineer, 16 Great Hollow Road, Hanover, NH, 03755, AIAA Member Grade for second author.

${ }^{3}$ Aerospace Technologist, 2101 NASA Parkway, Houston, TX, 77058/Mail Stop EC2, nonmember
} 


\section{Introduction}

$\square$ uture manned space exploration missions will need space suits with capabilities beyond the current state of $\mathbf{F}$ the art. Portable Life Support Systems (PLSSs) for these future space suits are extremely challenging, since they must maintain healthy and comfortable conditions inside the suit for long-duration missions while minimizing both weight and venting of consumables.

This paper describes an innovative system for temperature and humidity control in a space suit that promises to be simple, rugged, lightweight, and nonventing. Figure 1 shows the overall concept for the evaporation cooling/dehumidification garment (ECDG) and compares it with more conventional technology. The flexible ECDG absorbs both sensible heat (by conduction) and latent heat (by condensation). The garment provides cooling by evaporating water, which is then captured in a lithium chloride absorber/radiator (LCAR). The powerful LiCl desiccant in the LCAR maintains a low water vapor pressure that enables the ECDG to provide evaporative cooling at temperautres low enough for comfortable operation inside the space suit.

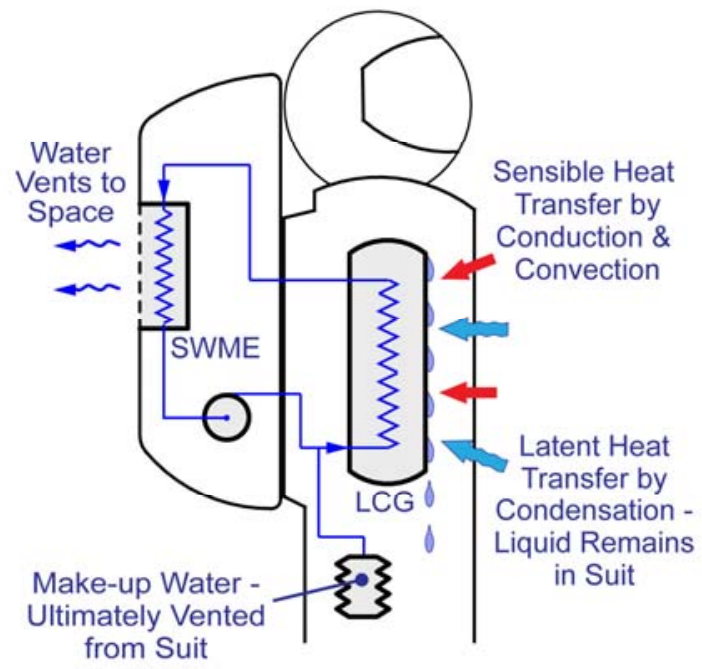

(a) Existing Technology Vents Cooling Water and Accumulates Perspiration Inside Suit

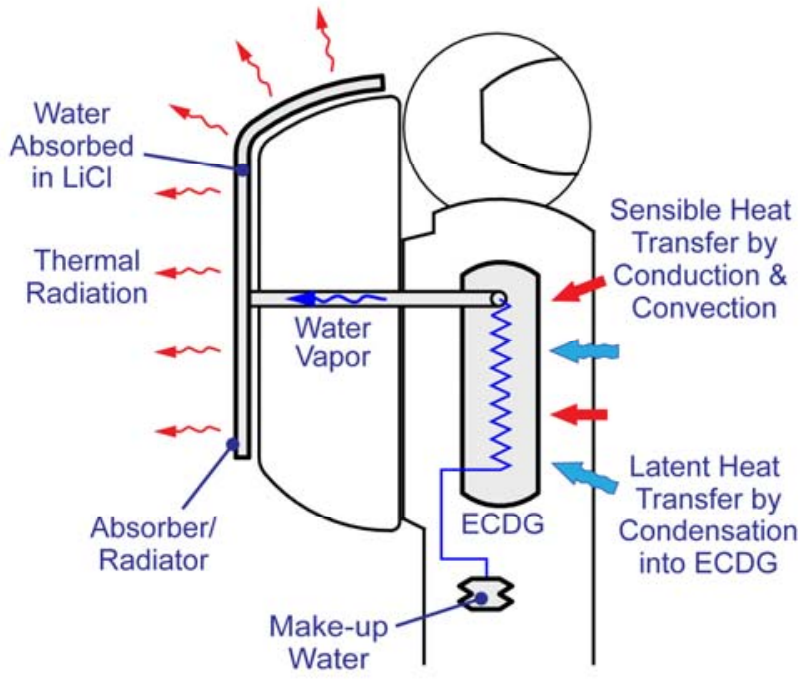

(b) The Proposed ECDG Vents Zero Water and Maintains a Cool, Dry Environment Inside Suit

Figure 1. Thermal and Humidity Control System Concept

\section{Design Requirements}

Human metabolism can generate large amounts of waste heat-over a horsepower under conditions of heavy exercise. To maintain a constant temperature, the body employs two cooling mechanisms: "sensible" heat transfer (i.e., conduction and convection of heat to the surrounding environment) and "latent" heat transfer (i.e., heat rejection by perspiration and expired water vapor). At rest, most heat rejection occurs via transfer of sensible heat, but at higher metabolic rates the latent heat contribution dominates. Figure 2 presents design data for crew member heat rejection as a function of activity level, pre- and post-exercise. ${ }^{4}$ Sensible heat rejection remains relatively constant over a wide range of activity levels, while the rate of latent heat rejection varies widely. After 30 minutes of exercise (defined as $75 \%$ of max $\mathrm{O} 2$ ventilation), latent heat rejection is more than three times greater than sensible heat rejection, and the water production rate is nearly $1 \mathrm{~L} / \mathrm{hr}$. Within the small volume of a space suit, these heat and humidity loads can quickly lead to highly uncomfortable conditions which can endanger the astronaut as performance degrades with increasing core temperature.

\footnotetext{
4 “Exploration Life Support Baseline Values and Assumptions Document,” NASA CR-2006-xxxxx
} 


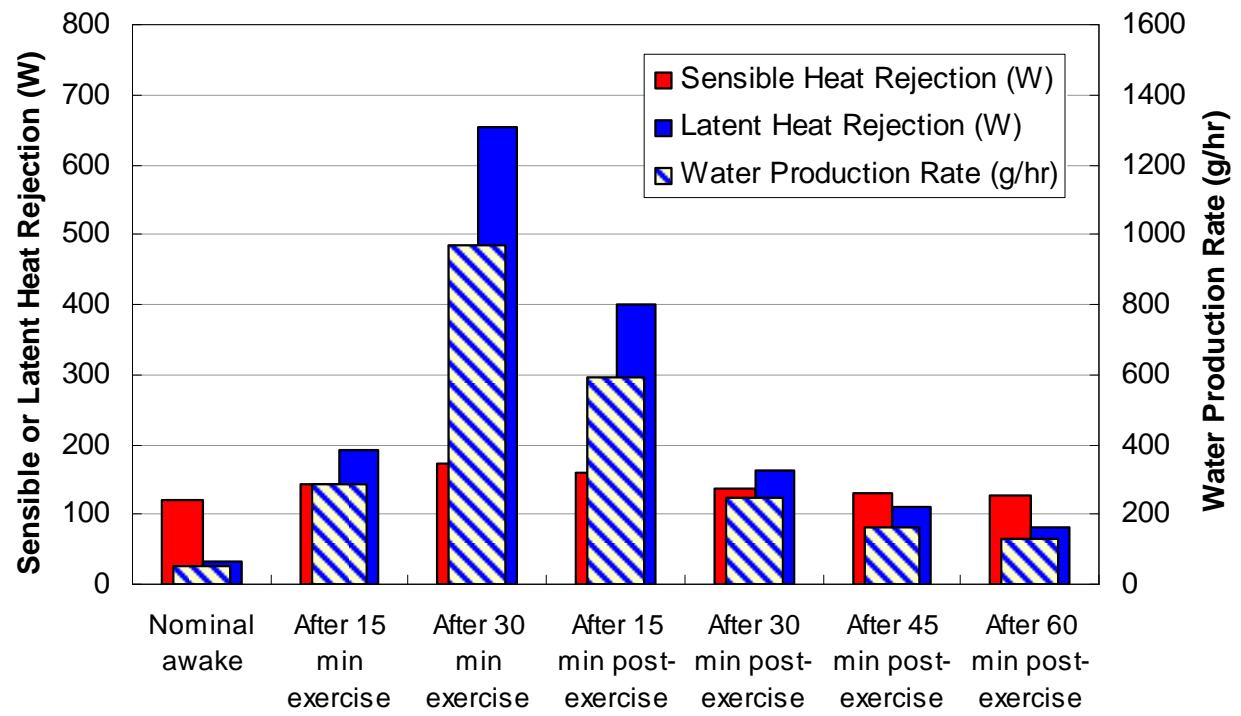

Figure 2. Heat Rejection Mechanisms

Table 1 lists the nominal design requirements for a space suit cooling system designed for an 8-hr EVA mission in a nominal lunar environment. ${ }^{5}$ Based on the data shown in Figure 2, we've assumed that, on average, half of the crew member's metabolic heat is rejected by perspiration. In this case, half the makeup water needed for a typical cooling garment can be eliminated by using an ECDG. Using our most recent size and mass estimates for the LCAR, the overall mass of the thermal control system is $6.1 \mathrm{~kg}$, providing an overall refrigeration density of $210 \mathrm{~W}$ $\mathrm{hr} / \mathrm{kg}$. More details on the thermal requirements and sizing calculations can be found in the papers ${ }^{6,7}$ that describe the LCAR.

\begin{tabular}{|c|c|}
\hline Cooling capacity & 1280 \\
\hline Cooling temperature $\quad\left({ }^{\circ} \mathrm{C}\right)$ & 19 \\
\hline Nominal heat sink temp $(\mathrm{K})$ & 250 \\
\hline Mission duration $\quad(\mathrm{hr})$ & 8.0 \\
\hline
\end{tabular}

\section{ECDG Design}

Figure 3 and Figure 4 illustrate the ECDG concept. Cooling is generated by evaporation of water from a porous, hydrophilic pad. The pad is sandwiched between a Nafion pocket and a hydrophobic ePTFE laminate. The Nafion pocket is a sealed volume that is coupled to the LCAR. The low water vapor pressure inside the pocket causes water to diffuse from the pad and through the Nafion, evaporating on the inner surface and producing cooling. The hydrophobic ePTFE prevents direct contact between the hydrophilic pad and the crew member, ensuring that only water vapor can flow into the pad and enabling the pad to absorb latent heat.

\footnotetext{
${ }^{5}$ Based on a heat balance calculation using EVASSAT yielding a net $160 \mathrm{~W}$ cooling requirement.

${ }^{6}$ Izenson, M., Chen, W. and Trevino, L., "Lightweight, Flexible, and Freezable Heat Pump/Radiator for EVA Suits,” 38th International Conference on Environmental Systems, Paper 08ICES-0312, July 2008.

${ }^{7}$ Izenson, M., Chen, W., Passow, C., Phillips, S. and Trevino, L., "Advanced Design Heat Pump/Radiator for EVA Suits,” SAE 2009 International Conference on Environmental Systems, Paper 2009-01-2406, July 2009.
} 


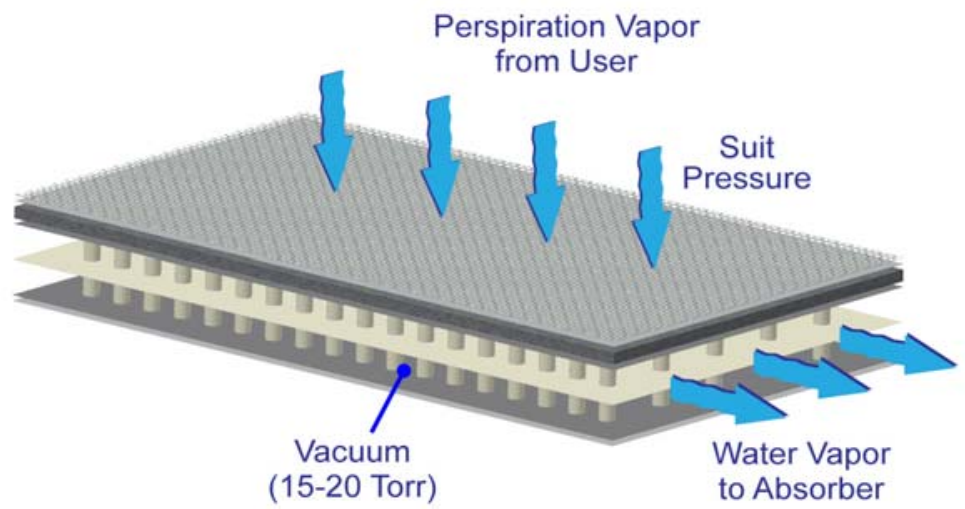

Figure 3. Operation of the Evaporation Cooling/Dehumidification Garment (ECDG)

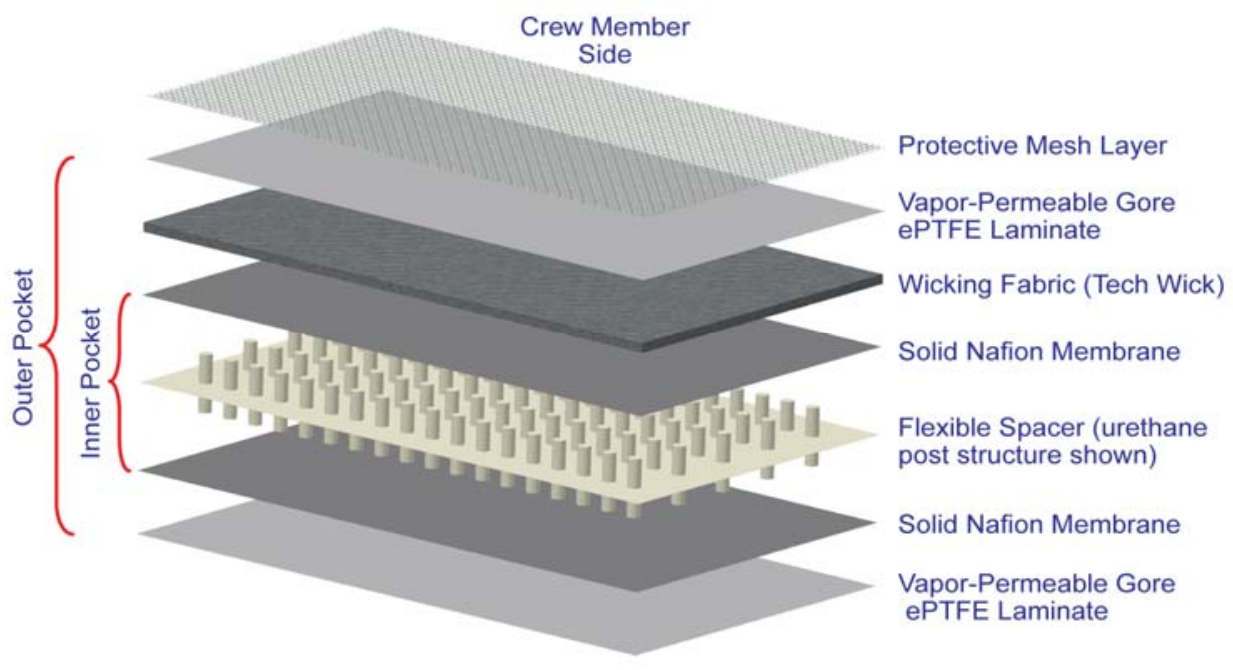

Figure 4. Construction of the ECDG

\section{ECDG Development and Testing}

Prototype ECDG. We developed methods to produce prototype ECDGs based on the basic design concept shown in Figure 4. The key fabrication processes are (1) a method to produce a flexible spacer that fits inside the Nafion pocket and maintains flow channels for water vapor, and (2) bonding and sealing methods for the Nafion sheets that surround the water vapor space. Figure 5 shows a typical prototype (with an area of $1 \mathrm{ft}^{2}$ ), which is also equipped with temperature and pressure instrumentation for separate effects testing. The vapor port is located in the center of the panel to ensure uniform water absorption across the panel area by minimizing the internal pressure differences due to vapor flow in the panel. In this test panel, we have inserted two pressure taps through the vapor port that enable us to measure the overall difference in vapor pressure from the corner of the pad to the center. A thermocouple is also inserted through the port to measure the internal temperature of the cooling panel. 


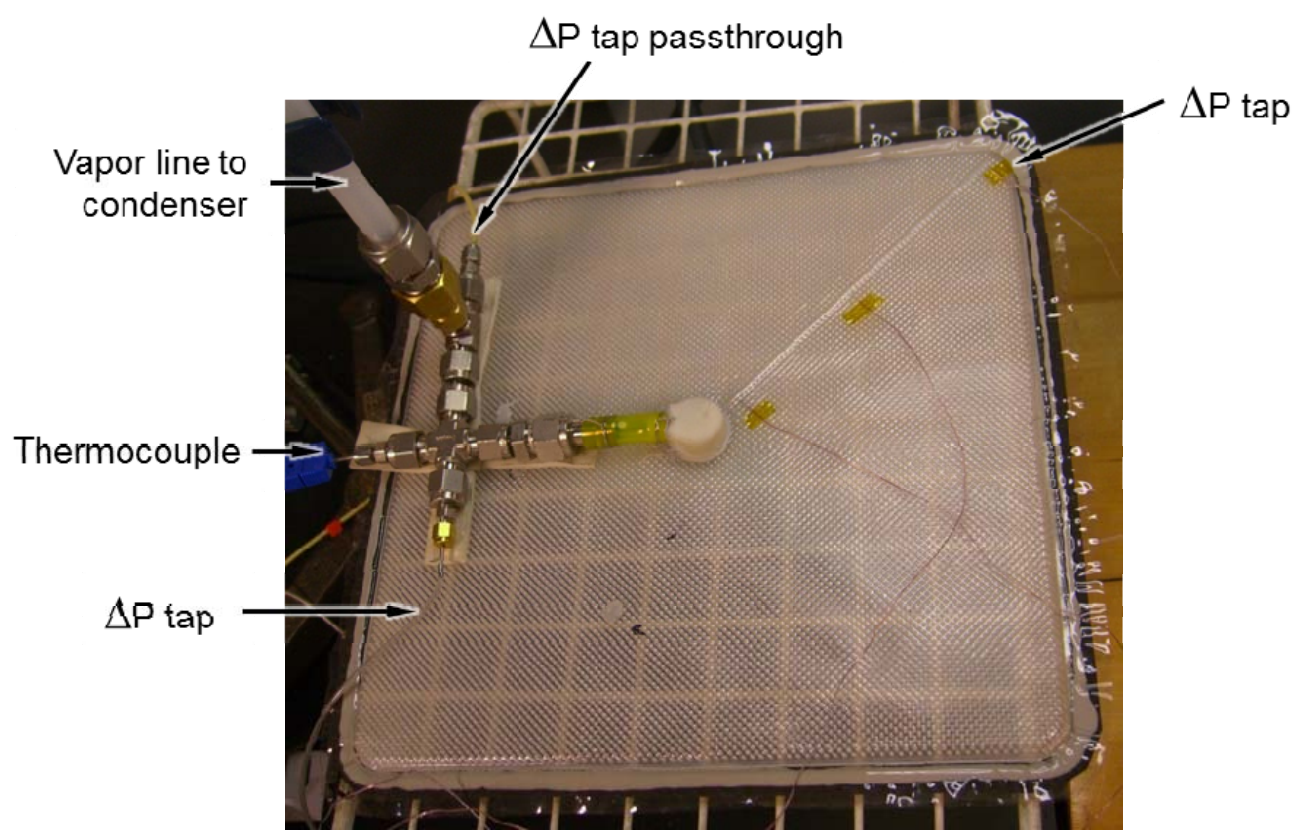

Figure 5. Prototype ECDG with Temperature and Pressure Instrumentation $(30 \mathrm{~cm} \times 30 \mathrm{~cm})$

Thermal Tests at Atmospheric Pressure. We measured the pouch performance of the prototype ECDG using a "sweating hot plate" test (Figure 6). The sweating hot plate comprises a wicking fabric backed by a heat spreader and film heater to simulate perspiration from the skin surface. The Nafion pouch was placed on top of the wicking material, separated by a $2 \mathrm{~mm}$-thick, hydrophobic mesh standoff. The wick material draws water from a reservoir to replace evaporated water. We measured both the mass of water removed from the reservoir and the heater power during the test to calculate the amount of heat dissipated by the pouch. To provide sensible cooling to the sweating hot plate, we installed a secondary wick on the surface of the pouch facing the simulated skin. Water was drawn directly to the secondary wick and evaporated to provide sensible cooling. This arrangement simulates the final version of the ECDG, in which we plan to deliver water to an array of water-permeable tubes inside the pouch.

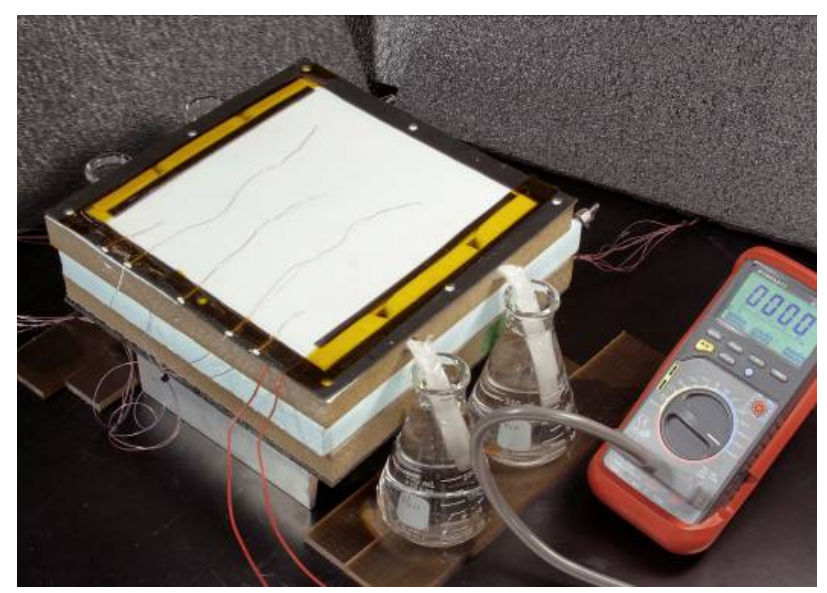

Figure 6. "Sweating Hot Plate" Simulates Sensible and Latent Heat Input to the ECDG

In the sweating hot plate tests, the pouch achieved a total cooling power of $39 \mathrm{~W}$ at an average skin temperature of $34.5^{\circ} \mathrm{C}$. We also measured the pouch performance without the secondary wick, which measured only the latent 
cooling potential of the pouch without supplemental sensible cooling. At 1 atmosphere pressure, the latent heat dissipation of the pouch was $18 \mathrm{~W}$ at an average skin temperature of $33.8^{\circ} \mathrm{C}$. These cooling powers are relatively low due to the high diffusion resistance of the 1-atm air gap between the simulated "skin" in the hot plate and the ECDG. Subsequent tests under simulated space suit pressure provided a more realistic diffusion resisance and demonstrated much higher cooling capacity.

Thermal Tests at Partial Vacuum Pressures. Figure 7 and Figure 8 show the test facility for measuring ECDG performance at space suit pressure $(4 \mathrm{psia})$. Eventually this test facility will incorporate a torso for more realistic testing of the ECDG, but this initial version of the system uses a flat geometry to simplify assessment of the fundamental phenomena. The facility incorporates the sweating hot plate to provide both sensible and latent heat to the ECDG. Water is fed to the wick in the sweating hot plate via a computer controlled syringe pump. We monitor the wick for appropriate water feed rate by checking for temperature uniformity of the skin surface and making sure that no water drips out of the wick. Figure 9 shows the temperatures measured at the evaporation surface and the pouch surface during these tests. At 4 psia, the pouch was able to absorb $65 \mathrm{~W}$ at an average skin temperature of $33.2^{\circ} \mathrm{C}$. We measured the latent heat dissipation by removing the secondary wick from the Nafion pouch surface and rerunning the test. Under this condition, the pouch absorbed $48 \mathrm{~W}$ at an average skin temperature of $34.1^{\circ} \mathrm{C}$.

Mass transfer resistance across the air gap between the skin surface and the cooling pouch depends on water vapor diffusion through the ambient air, and is therefore inversely proportional to the ambient pressure. As the ambient pressure decreases, the mass transfer resistance decreases and more water vapor can diffuse across the gap and reach the Nafion membrane surface. This results in higher cooling power, as shown in Figure 9 and Figure 10. Extrapolating from the results in Figure 10, as the ambient pressure decreases to a very small value, the mass transfer resistance in the air gap becomes negligible and the cooling power will reach about $75 \mathrm{~W}$. This value is consistent with the cooling power measured during tests in which we submerged the Nafion pouch in water. In these tests, in which the mass transfer resistance was zero, each Nafion coversheet provides about $75 \mathrm{~W}$ of cooling at $26^{\circ} \mathrm{C}$. 


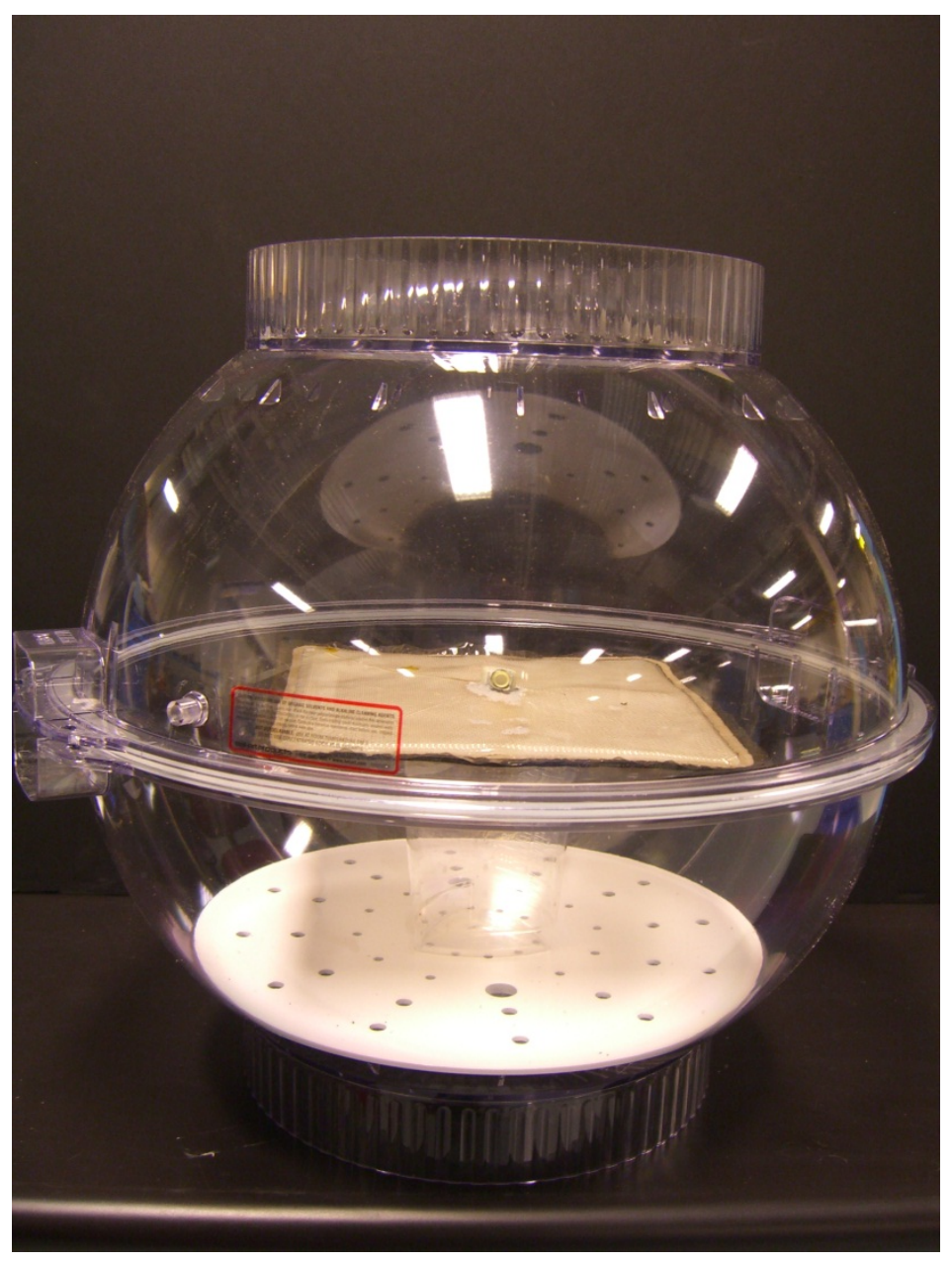

Figure 7. Vacuum Chamber for Testing ECDG Under Simulated Space Suit Conditions

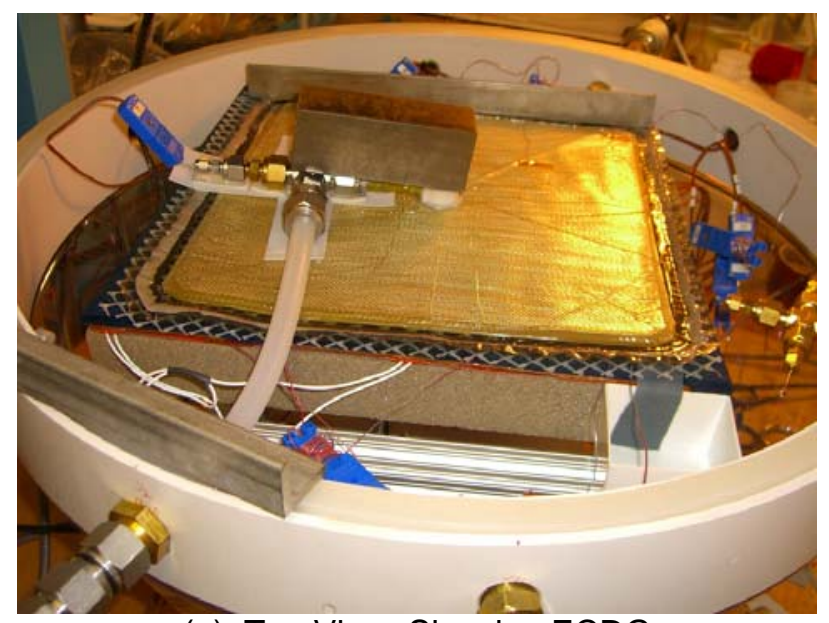

(a) Top View, Showing ECDG

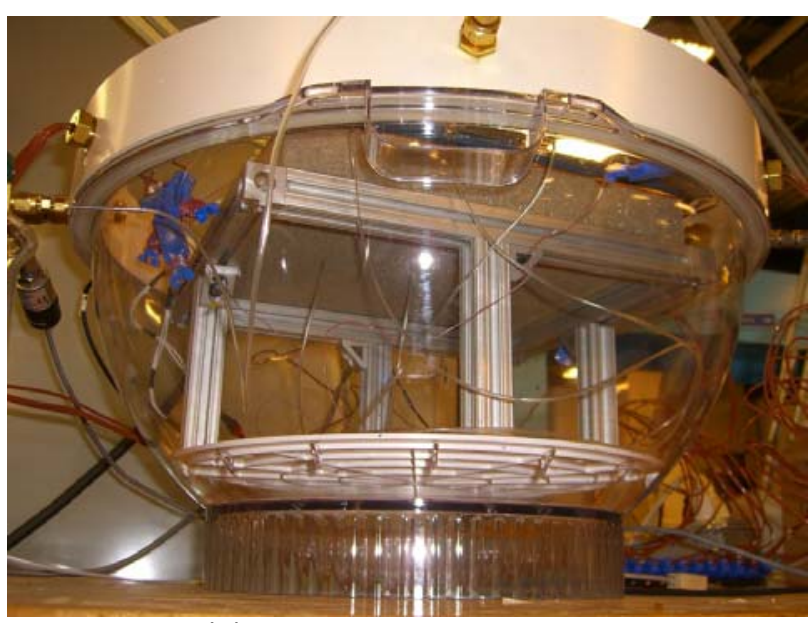

(b) View From Underneath

Figure 8. ECDG and Sweating Hot Plate Installed in Vacuum Chamber

American Institute of Aeronautics and Astronautics 


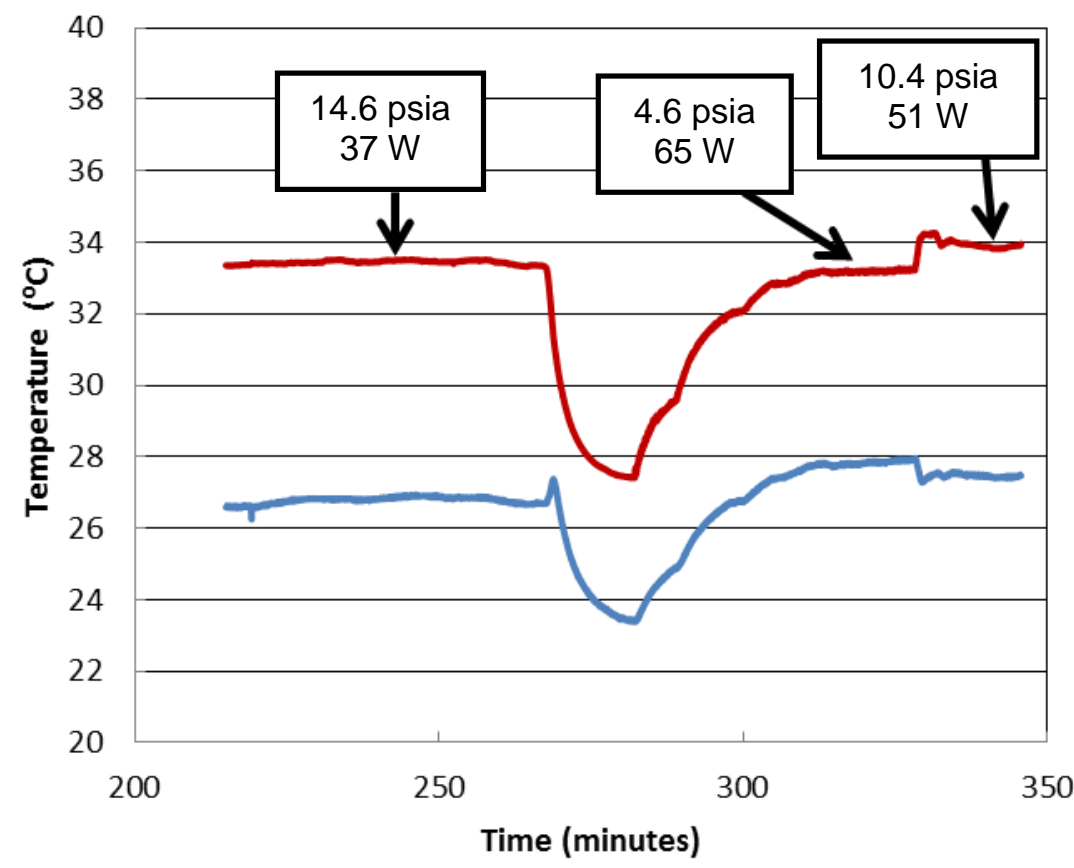

_ Average Skin Temperature

— Pouch Surface Temperature

Figure 9. Simulated Skin and Pouch Surface Temperatures Measured in the Integrated System Environmental Test Facility

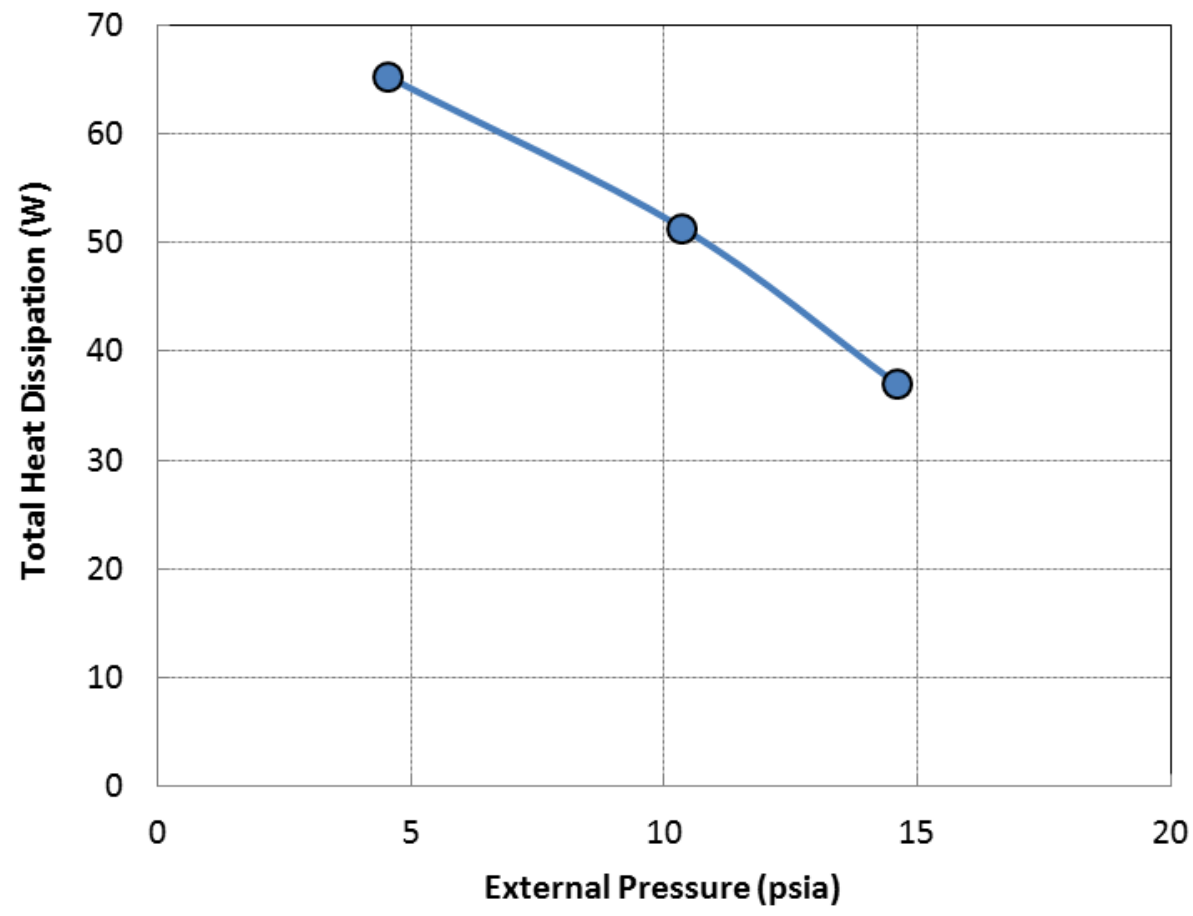

Figure 10. Total Heat Dissipation from a $1 \mathrm{ft} \times 1 \mathrm{ft}$ ECDG as a Function of External Pressure. The internal pressure was maintained at about 15 torr during these tests, corresponding to a saturation temperature of $17.5^{\circ} \mathrm{C}$ 
These data show that a full size ECDG with cooling surface area of $4 \mathrm{ft}^{2}$ will be able to provide $260 \mathrm{~W}$ of cooling, of which $192 \mathrm{~W}$ is latent heat. This is consistent with the average cooling power needed for a spacesuit. In the next reporting period, we plan to construct and test a pouch with a 0.002 inch-thick Nafion film in our Integrated System Environmental Test Facility to measure whether greater performance gains can be realized with the thinner membrane.

Internal Evaporation for Sensible Cooling. The first ECDG prototype provided cooling for sensible heat loads by evaporating water from an external wick. This wick was made of a highly permeable, nonwoven, hydrophilic material and would be supplied with water during operation from the space suit's reservoir of make-up water (Figure 1). During tests, this wick was cooled by vaporization of water from the internal surfaces of the Nafion pouch, and demonstrated high cooling rates in tests run at atmosphereic pressure and under simulated space suit pressure. However, it is difficult to control the amount of water supplied to the external wick. Highly permeable materials generally do not have a high enough capillary pumping capacity to be self regulating. Supplying water at positive pressure using a pump would require a complex feedback control system.

To simplify latent cooling, we have developed a concept for using hydrophobic hollow fibers to evaporate water inside the Nafion pouch. Arrays of hydrophobic hollow fibers with very high bubble points (typically 10 psia or greater) are available from commercial vendors. Figure 11 shows a commercial array of hollow fibers coupled to a flow manifold. Water pumped into the manifold will fill the hollow fibers but will not leak out if the internal pressure is less than the bubble point. If heat is applied to the array of fibers, water will evaporate through the pores. As long as the maximum pressure produced by the supply pump does not exceed the bubble point of the hollow fibers, only vapor will flow out of the fibers and the amount of makeup liquid supplied to the fibers will be regulated passively.

Figure 12 shows a test of flow uniformity, in which a manifolded sheet of hollow fibers was placed under a sheet of thermochromic paper, then pumped full of warm water. As the water fills the hollow fibers, the temperature rise changes the color of the thermochromic paper. The series of photographs in Figure 12 show that the warm water distributes very evenly across the array of hollow fibers.

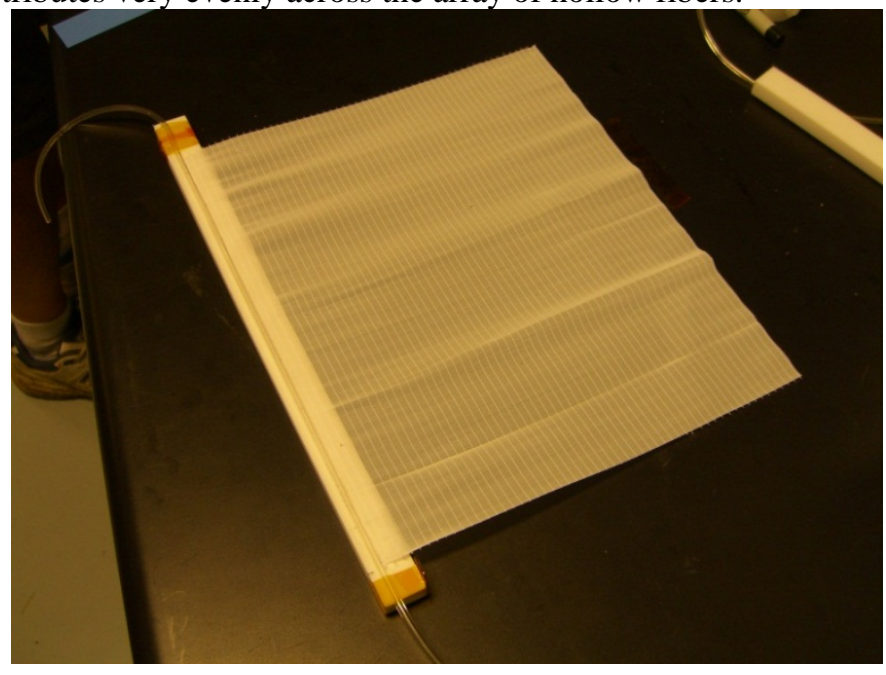

Figure 11. Hollow Fiber Array Couples with Flow Manifold

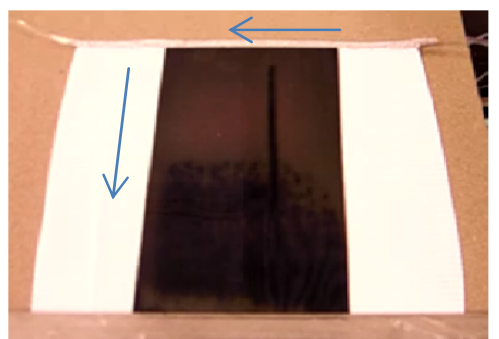

$t=0$ seconds

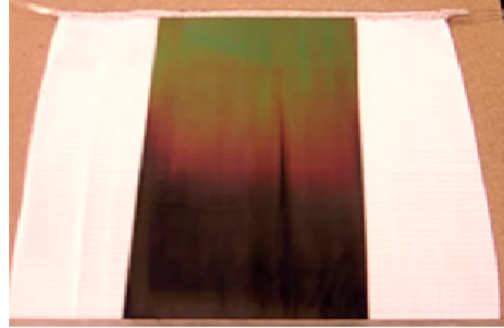

$t=10$ seconds

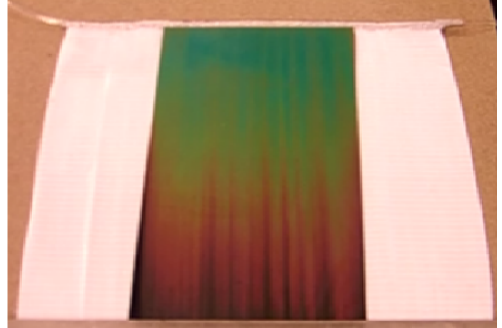

$\mathrm{t}=\mathbf{2 0}$ seconds

9

American Institute of Aeronautics and Astronautics 
Figure 12. Flow Uniformity Test of a Hollow Fib er Array Using Temperature-Sensitive Liquid Crystal Sheet. Black-red is coldest and green-Blue is warmest.

We have formulated a design concept and begun assembly of an ECDG that incorporates an internal array of hollow fibers to provide cooling for sensible heat loads. Figure 13 shows the design concept, in which a hollow fiber sheet is installed inside the Nafion pouch, between the flexible spacer and one of the outer Nafion sheets. The design includes dual manifolds for good water supply with flexible interconnects to allow the manifolds to move relative to each other for good flexibility and to accommodate expansion of the Nafion sheets. Figure 14 is a photograph of a hollow-fiber ECDG during assembly.

Flexible connection tubes to allow water vapor headers to

Water flow

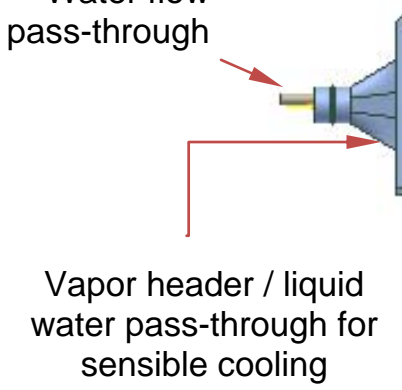
sensible cooling

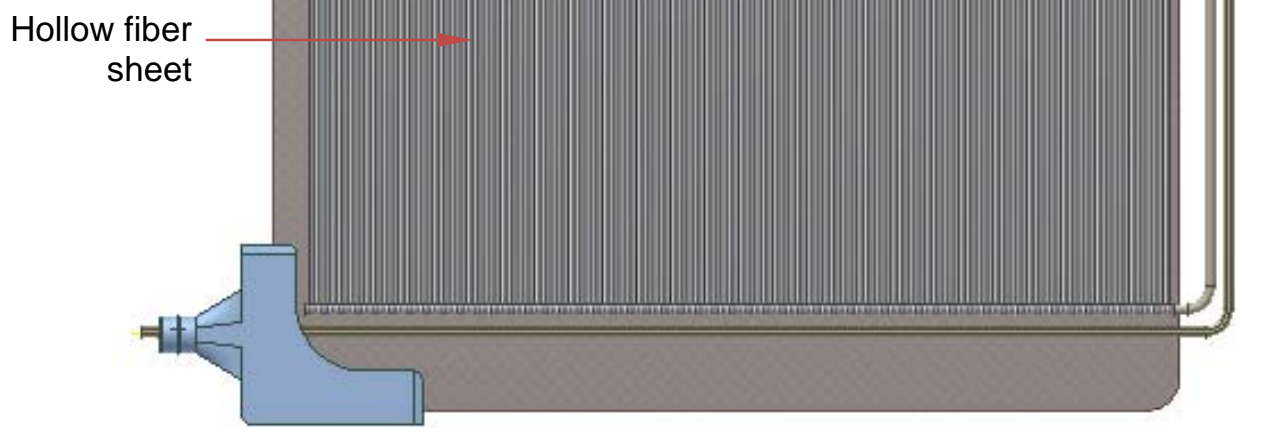

Figure 13. Conceptual Design of ECDG With Hollow Fiber Sheets for Supplemental Cooling 


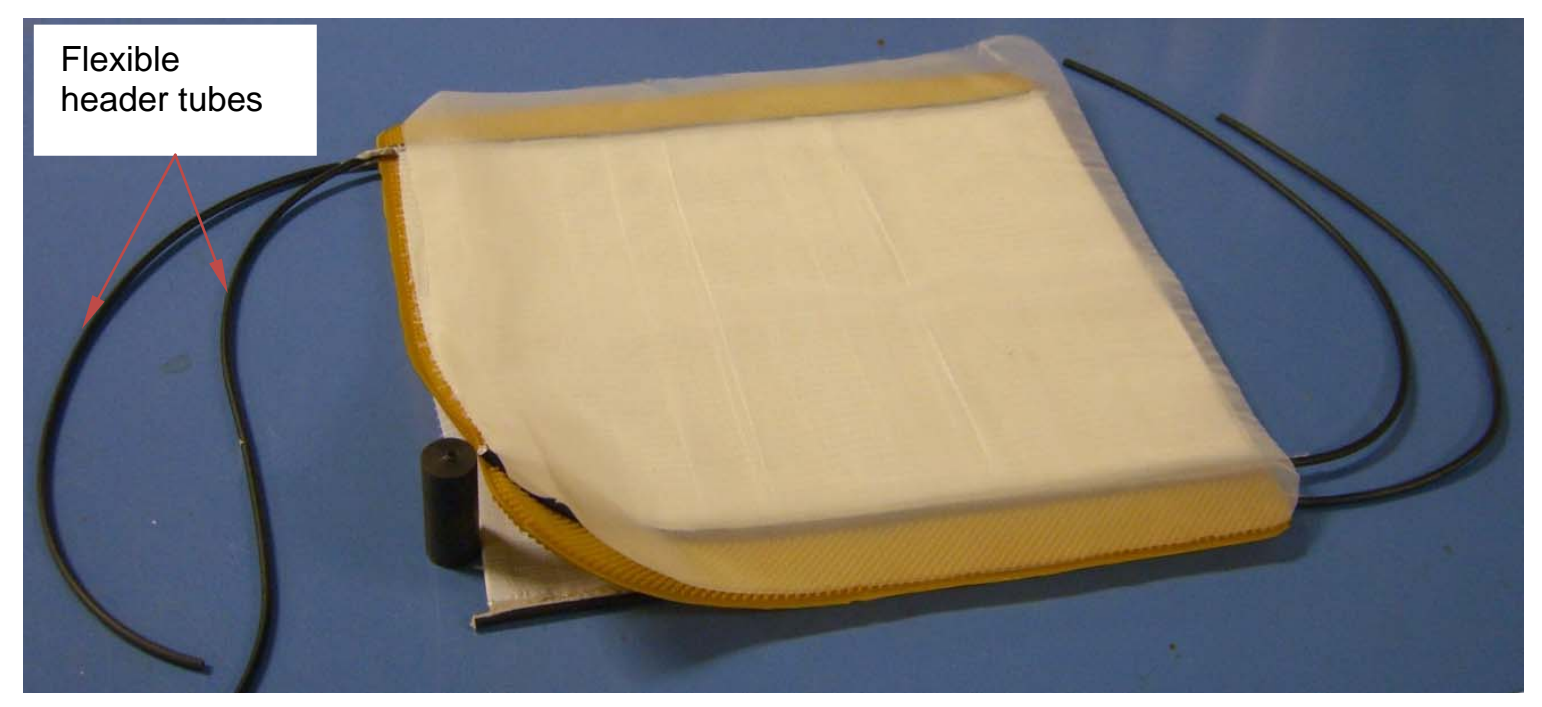

Figure 14. Post Field with Hydrophobic Hollow Fiber Sheets for Supplemental Cooling

\section{Conclusion}

Evaporative Coolign and Dehumidification Garment (ECDG) prototypes have been developed and tested under conditions that simulate operation in space suits. The ECDG is designed to operate with a lithium chloride absorber radiator (LCAR) as part of a nonventing thermal and humidity control system in future portable life support sytems. The ECDG prototypes have demonstrated a totcal cooling capacity of $65 \mathrm{~W} / \mathrm{ft}^{2}$ from simulated skin at a temperature of $33.2^{\circ} \mathrm{C}$. More than two-thirds of the cooling $\left(48 \mathrm{~W} / \mathrm{ft}^{2}\right)$ was latent cooling by direct absorption of water vapor. A $4 \mathrm{ft}^{2}$ cooling garment built using this technology should be able to provide enough cooling and denumidification for future space suits, providing a comfortable operating environment for the crew and venting almost no water. A new design with imporved sensible cooling is under development based on an internal array of hydrophic hollow fibers. Preliminary tests of these fibers show high flow capacity and excellent flow uniformity.

\section{Acknowledgments}

Work reported in this paper was sponsored by the Crew and Thermal Systems Division at NASA Lyndon B. Johnson Space Center through a Phase I Small Business Innovation Research contract. 